\title{
Introdução de alimentos complementares nos primeiros dois anos de vida de crianças de escolas particulares no município de São Paulo
}

\author{
Introduction of complementary foods in the first two years of life of children attending private schools in \\ the city of São Paulo, Brazil
}

Viviane Gabriela N. Simon ${ }^{1}$, José Maria P. de Souza², Sonia Buongermino de Souza ${ }^{3}$

\section{RESUMO}

Objetivos: Verificar a idade de introdução de alimentos complementares nos primeiros dois anos de vida e sua relação com variáveis demográficas e socioeconômicas de crianças matriculadas em pré-escolas particulares do município de São Paulo.

Métodos: Estudo transversal com informações demográficas e socioeconômicas de 566 crianças, sendo verificada a idade em meses de introdução dos alimentos complementares. Foi considerada como variável dependente a idade em meses da introdução dos alimentos complementares e, como variáveis independentes ou explanatórias, a idade e escolaridade maternas, a condição de trabalho materno e a renda familiar. Para análise da relação entre as variáveis, utilizou-se a técnica de regressão múltipla de Cox.

Resultados: $50 \%$ das crianças eram do sexo masculino e $61 \%$ maiores de 4 anos. A maior proporção das mães tinha nível superior de escolaridade e trabalhava fora. A renda familiar mostrou uma população de alto nível socioeconômico. A água e/ou chá, frutas e leite não-materno foram introduzidos antes do sexto mês de vida. A variável 'idade da mãe' mostrou associação com introdução de três grupos de alimentos: cereais, carne e guloseimas.

Conclusão: Alimentos complementares foram introduzidos precocemente nessa população de nível socioeconômico elevado e a única variável que se associou à introdução desses alimentos foi a idade materna.

Palavras-chave: suplementação alimentar; pré-escolar; nutrição do lactente; idade materna.

\section{ABSTRACT}

Objective: To verify the age of complementary food introduction in the first two years of life and its relation to demographic, social, and economic status of preschool children of private schools in the city of São Paulo, Brazil.

Methods: Cross-sectional survey with demographic, social and economic status information. The studied population included 566 children. The age in months of complementary foods introduction was verified. The dependent variable was the age in months of complementary foods introduction. Independent or explanatory variables were: mother's age and education, maternal work conditions and household income. The relation between variables was analyzed by Cox multiple regression.

Results: $50 \%$ of the children were males and $61 \%$ were older than 4 years old. Most mothers had higher education and were employed. The household income showed a population of high social and economic level. Water and/ or tea, fruits and non-breast milk were introduced before the sixth month of life. The variable 'maternal age' showed association with the introduction of three groups of foods: cereals, meat and junk food.

Conclusion: Early complementary food introduction occurred in this population of high socioeconomic level, and the only variable associated with this occurrence was maternal age.

Key-word: supplementary feeding; child, preschool; infant nutrition; maternal age.
Instituição: Faculdade de Saúde Pública da Universidade de São Paulo (USP), São Paulo, SP, Brasil

${ }^{1}$ Bolsista de Pós-Doutorado da Fundação de Amparo a Pesquisa do Estado e São Paulo (Fapesp) junto ao Departamento de Saúde Materno-Infantil da Faculdade de Saúde Pública da Universidade de São Paulo, São Paulo, SP, Brasil 2Professor titular do Departamento de Epidemiologia da Faculdade de Saúde Pública da USP, São Paulo, SP, Brasil

${ }^{3}$ Professora doutora do Departamento de Nutrição da Faculdade de Saúde Pública da USP, São Paulo, SP, Brasil
Endereço para correspondência:

Viviane Gabriela Nascimento Simon

Avenida Doutor Arnaldo, 715

CEP 01246-904 - São Paulo/SP

E-mail: vivianesimon@usp.br

Recebido em: 4/12/08

Aprovado em: 31/5/09 


\section{Introdução}

$\mathrm{O}$ aleitamento materno representa a forma mais natural e apropriada de alimentar uma criança nos primeiros meses de vida, provendo crescimento e desenvolvimento adequados ${ }^{(1)}$. $\mathrm{O}$ aleitamento materno propicia outros benefícios, como aumento da imunidade da criança e proteção contra as infecções gastrintestinais e respiratórias ${ }^{(2)}$, proteção contra as doenças metabólicas como o diabetes ${ }^{(3)}$, além de benefícios econômicos para a família e sociedade ${ }^{(4)}$.

A introdução precoce de alimentos complementares, ou seja, antes dos seis meses de vida, aumenta a morbimortalidade infantil como consequência de menor ingestão dos fatores de proteção existentes no leite materno e exposição a patógenos ${ }^{(5)}$. Sob o ponto de vista nutricional, a introdução precoce dos alimentos complementares, por ocasionar a diminuição de ingestão de leite materno, pode ser desvantajosa. Introduzir os alimentos complementares tardiamente também é desfavorável, porque o crescimento da criança se torna mais lento e o risco de desnutrição e de deficiência de micronutrientes aumenta ${ }^{(6)}$.

Os alimentos complementares são representados, habitualmente, pelos cereais, frutas, legumes, verduras e raízes, além das carnes e ovos, e devem ser oferecidos a partir dos seis $\operatorname{meses}^{(7)}$. Entretanto, observações referentes ao padrão alimentar nos dois primeiros anos de vida têm mostrado que a alimentação é predominantemente láctea, mesmo após os seis meses, preparada à base de leite de vaca em pó ou líquido, acrescida de farináceo e açúcar ${ }^{(8,9)}$.

Alguns estudos demonstram que a introdução precoce da a limentação complementar está associada à baixa renda familiar, pouca idade materna, primiparidade e retorno da mãe ao trabalho, baixa escolaridade materna e maternidade precoce ${ }^{(10,11)}$.

A partir do exposto, buscou-se verificar a idade de introdução de alimentos complementares nos primeiros dois anos de vida e sua relação com variáveis demográficas e socioeconômicas de crianças de 2 a 6 anos de idade matriculadas em escolas particulares do município de São Paulo.

\section{Métodos}

Este estudo é parte da pesquisa intitulada 'Relação do aleitamento materno, alimentação complementar e obesidade e sobrepeso em crianças de 2 a 6 anos de idade matriculadas em escolas particulares no Município de São Paulo'.

O estudo é transversal, com crianças de dois a seis anos de idade completos, matriculadas em escolas particulares, localizadas no bairro de Santana, na zona norte do município de São Paulo. Para maior facilidade e operacionalidade no desenvolvimento desta pesquisa, foi realizado contato telefônico com sete escolas particulares, que concordaram em participar do estudo.

O período de coleta de dados foi de agosto a novembro de 2004 e de março a maio de 2005. Devido à dificuldade da presença da mãe e/ou responsável durante o período de aula da criança, os formulários com instruções para o autopreenchimento foram entregues pela escola para serem respondidos pelas próprias mães e/ou responsáveis e devolvidos posteriormente na escola. Foram enviados 809 questionários, dos quais $30 \%$ não foram devolvidos.

Todos os formulários foram analisados após serem devolvidos pelas escolas; para as informações incompletas ou nãocompreendidas, utilizou-se contato telefônico diretamente com a mãe e/ou responsável, para obtenção das informações corretas, não havendo perdas. Fizeram parte do estudo 566 crianças.

Foi verificada a idade em meses da introdução dos seguintes alimentos complementares: água e/ou chá, leite não-materno (leite em pó comum, leite formulado, leite fluido), frutas (suco de fruta, papinha de fruta ou fruta in natura), açúcar e/ou mel, espessantes, cereais e tubérculos, hortaliças (verduras e legumes), feijão, carne bovina, frango, peixe, gema de ovo, ovo inteiro, embutidos, achocolatados, guloseimas (bala e/ou pirulito, chocolate e outros alimentos industrializados). Foram considerados, para cada criança, apenas os alimentos introduzidos nos primeiros dois anos de vida. Foram coletadas informações sobre idade e escolaridade materna, condição de trabalho materno e renda familiar.

$\mathrm{Na}$ análise dos dados, foi considerada como variável dependente a idade de introdução em meses dos alimentos complementares. Os alimentos foram estudados em oito grupos: água e/ou chá, leite não materno, frutas, açúcar (açúcar e/ou mel e espessantes), cereais (cereais e tubérculos e hortaliças), feijão, carnes (bovina, frango, peixe, gema de ovo, ovo inteiro, embutidos) e guloseimas (achocolatados, bala e/ ou pirulito, chocolate, outros alimentos industrializados). $\mathrm{O}$ critério utilizado para formar os grupos de análise foi a idade semelhante de introdução dos alimentos complementares na alimentação da criança.

As variáveis independentes ou explanatórias foram: idade materna ( $\leq 35$ anos e $>35$ anos), escolaridade materna (ensino fundamental, ensino médio e ensino superior), condição de trabalho materno (trabalha fora ou não) e renda 
familiar. Para a análise, decidiu-se utilizar as variáveis de forma dicotômica e, para a idade materna e renda familiar, as categorias foram obtidas adotando-se a mediana como ponto de corte.

A caracterização da amostra é apresentada por meio de distribuições de frequências. A introdução dos alimentos, é mostrada em curvas segundo a técnica de Kaplan-Meier ${ }^{(12)}$, permitindo analisar os dados com se fossem prospectivos, apesar de coletados transversalmente. Para a análise da relação entre idade de introdução de cada grupo de alimentos complementares e as variáveis independentes, utilizou-se a técnica de regressão múltipla de Cox, adotando-se o critério $p<0,05$ para permanência da variável no modelo final. São apresentados os $p$ descritivos e os intervalos com $95 \%$ de confiança. As análises estatísticas foram executadas com o aplicativo Stata 10.1.

Todas as mães e/ou responsáveis pelas crianças concordaram com a participação na pesquisa e assinaram um Termo de Consentimento Livre e Esclarecido. O presente estudo foi aprovado pelo Comitê de Ética em Pesquisa da Faculdade de Saúde Pública da Universidade de São Paulo.

\section{Resultados}

A amostra foi composta por $50,3 \%$ de crianças do sexo masculino; 61,3\% eram maiores de 4 anos de idade; 52,8\% das mães tinham idade menor ou igual a 35 anos e a maior proporção delas mostrava nível superior de escolaridade $(84,6 \%)$. Em relação ao trabalho materno, $73,5 \%$ trabalhavam fora. A mediana da renda familiar foi $\mathrm{R} \$ 6.450,00$ (18,9\% das famílias estudadas não quiseram informar sua renda), caracterizando uma população de nível socioeconômico elevado.

Nas Figuras 1, 2 e 3, observa-se a introdução precoce de quase todos os grupos de alimentos estudados. A Figura 1 mostra os três grupos de alimentos com menores idades medianas de introdução, antes dos seis meses. Os grupos de alimentos com idade mediana de introdução entre seis e dez meses foram as carnes, cereais e feijão, apresentados na Figura 2. Na Figura 3 os grupos do açúcar e guloseimas apresentaram maiores idades medianas, entre nove e 12 meses.

Nos oito modelos finais da análise múltipla, segundo a regressão de Cox evidenciada na Tabela 1, a única variável associada à introdução dos alimentos complementares foi a idade da mãe; mesmo assim, apenas para três grupos de alimentos: cereais, carnes e guloseimas. Verifica-se que mães

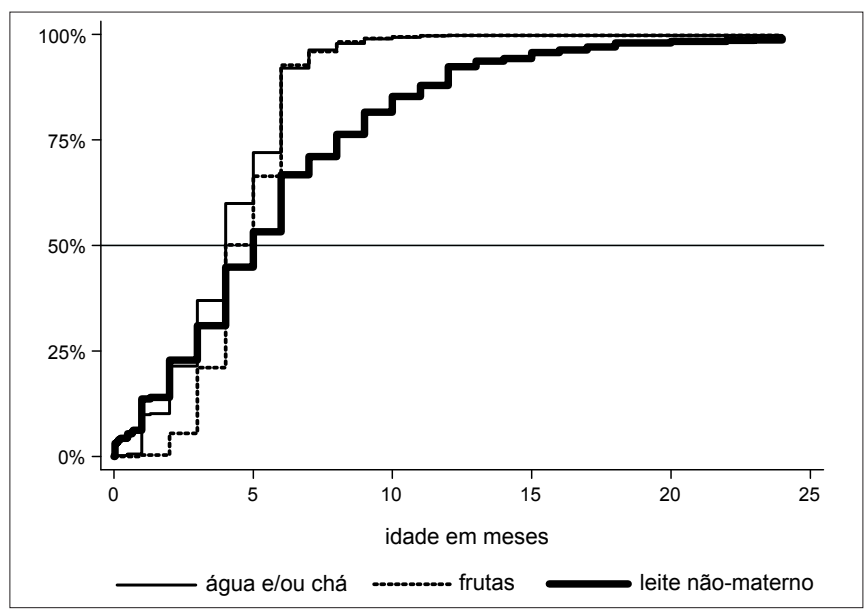

Figura 1 - Proporção de crianças que já tiveram a introdução dos grupos de alimentos 'água e/ou chá', 'frutas' e 'leite nãomaterno'.

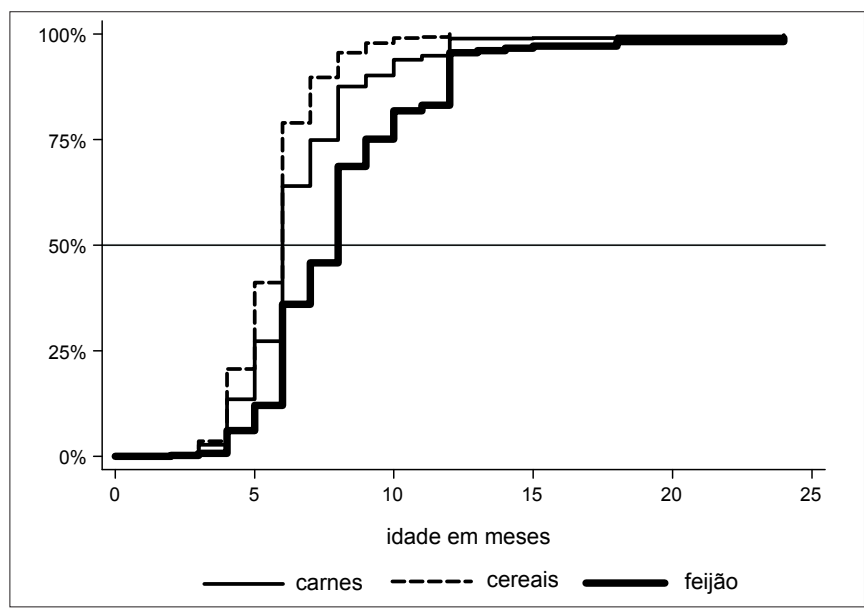

Figura 2 - Proporção de crianças que já tiveram a introdução dos grupos de alimentos 'carnes', 'cereais' e 'feijão'.

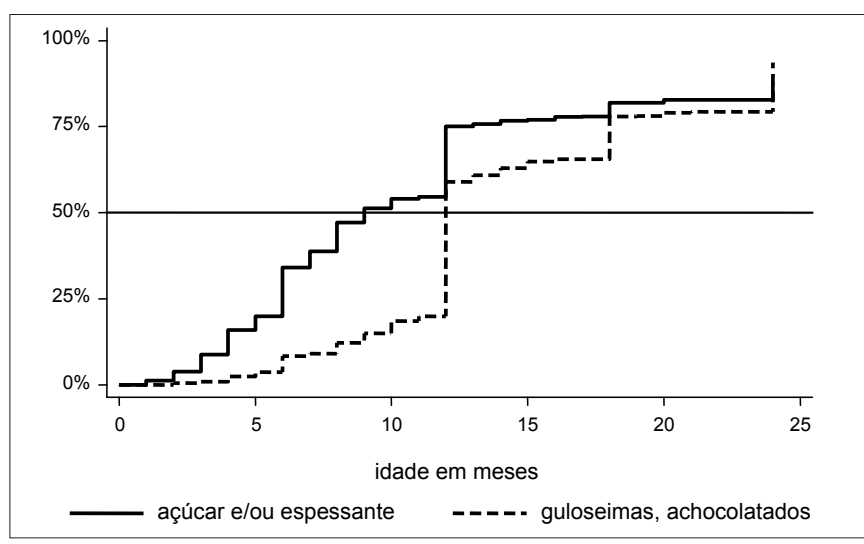

Figura 3 - Proporção de crianças que já tiveram a introdução dos grupos de alimentos 'açúcar e/ou espessante'e 'guloseimas, achocolatados'. 
Tabela 1 - Resultados dos modelos finais da análise de Cox

\begin{tabular}{|c|c|c|c|c|}
\hline \multirow{2}{*}{ Variável } & \multicolumn{3}{|c|}{ Cereais } & \multirow{2}{*}{ IC95\% } \\
\hline & HR & $\mathbf{Z}$ & Valor de $p$ & \\
\hline \multicolumn{5}{|l|}{ Idade da mãe } \\
\hline$\leq 35$ anos & 1 & & & \\
\hline \multirow[t]{3}{*}{$>35$ anos } & 1,18 & 1,97 & 0,04 & $1,00-1,40$ \\
\hline & & Carnes & & \multirow{2}{*}{ IC95\% } \\
\hline & HR & $\mathbf{Z}$ & Valor de $p$ & \\
\hline \multicolumn{5}{|l|}{ Idade da mãe } \\
\hline$\leq 35$ anos & 1 & & & \\
\hline \multirow[t]{3}{*}{$>35$ anos } & 1,24 & 2,52 & 0,01 & $1,05-1,46$ \\
\hline & & Ioseim & & \multirow{2}{*}{ IC95\% } \\
\hline & HR & Z & Valor de $p$ & \\
\hline \multicolumn{5}{|l|}{ Idade da mãe } \\
\hline$\leq 35$ anos & 1 & & & \\
\hline$>35$ anos & 0,84 & $-2,19$ & 0,03 & $0,69-0,98$ \\
\hline
\end{tabular}

HR: Hazard Ratio (razão de taxas de introdução); Z: escore z na curva normal; IC: intervalo com 95\% de confiança

mais velhas introduziram mais cedo cereais e carnes e introduziram mais tarde as guloseimas.

\section{Discussão}

A interrupção precoce do aleitamento materno continua a acontecer, apesar das evidências de que o leite materno, oferecido de forma exclusiva, atende às necessidades de nutrientes e de energia do lactente até cerca de seis meses de vida.

A introdução de alimentos complementares frequentemente ocorre no período de zero a seis meses de idade, período este em que, segundo a Organização Mundial da Saúde ${ }^{(13)}$, a criança deveria receber apenas o leite materno, sem necessidade de nenhum outro alimento. A água e/ou chá e a fruta apresentaram, neste estudo, idade mediana de introdução de quatro meses, e o leite não-materno, de cinco meses, caracterizando, portanto, sua introdução precoce. As idades medianas de introdução observadas para carnes, cereal e tubérculos, feijão, açúcar e guloseimas ocorreram a partir de seis até 12 meses.

Estudo realizado no Centro-Oeste brasileiro mostrou consumo de água e chá superior a $20 \%$, logo ao nascer. No primeiro mês, o uso de chá foi mais frequente que o de água; entretanto, em torno dos 30 dias, a água passou a ser mais consumida que o chá. $\mathrm{O}$ terceiro alimento referido foi o leite de vaca, em pó ou fluido. As frutas também foram introduzidas precocemente, sendo iniciadas aos três meses de idade ${ }^{(14)}$.

No presente estudo, não foi verificado o tipo de leite nãomaterno introduzido na alimentação da criança; porém, como a população estudada faz parte de um nível socioeconômico alto, acredita-se que a maioria das crianças deve ter recebido fórmulas infantis, oferecidas no mercado a preços altos, sendo sua aquisição restrita a apenas parte da população. Segundo a Sociedade de Pediatria de São Paulo ${ }^{(15)}$, na impossibilidade de aleitamento materno, as fórmulas devem ser introduzidas na alimentação. A escolha do tipo de leite desempenha um papel muito importante sobre a densidade energética na $\operatorname{alimentação~}^{(16)}$.

Simon et $a l^{(17)}$, em estudo de coorte realizado em Hospital Universitário do Município de São Paulo, analisaram 326 crianças. Os autores verificaram que os primeiros grupos de alimentos a serem introduzidos são água e chá, leite nãomaterno e frutas, sendo a idade mediana de introdução de 28 , 60, e 106 dias, respectivamente. Verduras e legumes, cereais e tubérculos também tiveram idade mediana de introdução antes do sexto mês (137 e 150 dias, respectivamente). Para carne, feijão e ovo, as medianas foram maiores do que seis meses. O estudo citado mostra os mesmos alimentos introduzidos para iniciar a alimentação complementar, porém em ordem diferente e medianas inferiores às do presente estudo.

Na cidade de Feira de Santana (BA), com 2.319 crianças de famílias com renda entre um e dois salários mínimos, Vieira et $a l^{(18)}$ verificaram que, no primeiro mês de vida, as crianças amamentadas já ingeriam água $(7,3 \%)$, chás $(23,2 \%)$, sucos $(2,7 \%)$, papas de frutas $(0,7 \%)$ e papas de legumes $(2,0 \%)$, o que mostra a precocidade não só da introdução de água, chá ou suco, mas também de outros alimentos. Outro estudo realizado na região do Alto Jequitinhonha (MG) em 2000, 
com população de baixo poder aquisitivo, verificou que, com menos de um mês de vida, 33,6\% das crianças já recebiam chás; $12,4 \%$, água; $2,7 \%$, suco; $8,0 \%$, leite não-materno; e $0,9 \%$, sopinhas ${ }^{(19)}$. Esses dados mostram que, em população de nível socioeconômico mais baixo, a introdução de alimentos também ocorre de maneira precoce.

A maioria das pesquisas realizadas para verificar a alimentação da criança é transversal, como este estudo, o que facilita a comparação. Parece não haver dúvida de que realmente os alimentos responsáveis pela interrupção do aleitamento materno exclusivo são: água e/ou chá, frutas e leite não-materno. Verifica-se grande consumo de água e/ou chá precocemente na alimentação da criança, fortalecendo a convicção de que, quando a mãe oferece água e/ou chá à criança, ela não tem como objetivo a nutrição, mas sim a medicação, atendendo muitas vezes a fatores culturais e costumes antigos ${ }^{(20)}$. Observa-se, com isso, que ainda ocorre a desinformação da população a respeito da introdução de água e chá na dieta da criança

Em relação às associações entre idade de introdução dos alimentos complementares e as variáveis socioeconômicas e demográficas, Saldiva et al ${ }^{(21)}$, em estudo transversal no qual foram analisados os dados do Projeto Amamentação e Municípios de 2004, conduzido em 136 municípios com 24.448 crianças, verificaram que o consumo de refeições lácteas estava associado ao fato de a mãe trabalhar fora e ter escolaridade superior a oito anos.

Estudo sobre a alimentação do lactente em duas comunidades rurais brasileiras e a relação com variáveis como educação da mãe e renda per capita da família observou que, quanto maior a renda per capita da família, maior é o consumo de alimentos industrializados; também se observou que a educação da mãe pode interferir no tipo de alimento oferecido à criança na alimentação complementar ${ }^{(22)}$.

Simon $e t$ al ${ }^{(17)}$ observaram associação estatisticamente significante entre mães com nível superior de escolaridade e a introdução mais tardia de água ou chá, o que sugere que a falta de informação é fator importante para introdução de líquidos não-nutritivos. $\mathrm{O}$ fato da mãe não trabalhar fora levou a uma introdução tardia de leite não-materno, o que demonstra que a mãe que fica com a criança em casa tem maior disponibilidade para amamentação. Já a introdução de carnes estava relacionada a níveis mais elevados de renda; essa associação pode estar relacionada ao custo elevado do alimento.

Outro estudo, ao investigar as características do desmame precoce no distrito noroeste da cidade de Campinas (SP), em 2001, com 385 crianças de baixo nível socioeconômico, mostrou associação entre desmame precoce e tempo de estudo materno: mães com menor tempo de estudo tendem a desmamar antes dos seis meses de idade ${ }^{(23)}$, ou seja, oferecem alimentos complementares precocemente.

Neste estudo, entretanto, observou-se que a única variável que apresentou associação significante com a idade de introdução de alimentos complementares foi a idade da mãe. As mães com idade superior a 35 anos introduziram mais cedo, na alimentação das crianças, cereal e carnes. As guloseimas foram introduzidas mais tarde, supondo que essas mães fossem mais experientes, provavelmente com mais de um filho, e que possuíssem maior consciência da importância da alimentação saudável, deixando as guloseimas para serem introduzidas por volta do final do primeiro ano de vida.

As variáveis 'escolaridade da mãe', 'condição de trabalho da mãe' e 'renda familiar' não apresentaram associação com a idade de introdução de alimentos complementares, talvez devido à homogeneidade do grupo estudado, o que pode ser considerado um aspecto limitante do estudo. Outra limitação foi o nível socioeconômico elevado, dificultando comparações com a maioria dos estudos, que foram realizados em população de baixo nível socioeconômico.

Pode-se concluir que, nessa população de nível socioeconômico elevado, os alimentos complementares foram introduzidos precocemente, com destaque para água e/ou chá, frutas e leite não-materno. A única variável que se associou à introdução dos alimentos foi a idade materna.

\section{Referências bibliográficas}

1. Kramer MS, Chalmers B, Hodnett ED, Sevkovskaya Z, Dzikovich I, Shapiro $S$ et al. Promotion of breastfeeding intervention trial (PROBIT): a randomized trial in the Republic of Belarus. JAMA 2001;285:413-20.

2. Oddy WH, Sly PD, de Klerk NH, Landau LI, Kendall GE, Holt PG et al. Breast feeding and respiratory morbidity in infancy: a birth cohort study. Arch Dis Child 2003;88:224-8.
3. Gimeno SG, Souza JM. Insulin-dependent diabetes mellitus and milk consumption: a case-control study in São Paulo, Brasil. Diabetes Care 1997;20:1256-60.

4. Araújo MF, Del Fiaco A, Pimentel LS, Schmitz BA. Custo e economia da prática do aleitamento materno para a família. Rev Bras Saude Matern Infant 2004;4:135-41. 
5. Dewey KG, Cohen RJ, Brown KH, Rivera LL. Effects of exclusive breastfeeding for four versus six months on maternal nutritional status and infant motor development: results of two randomized trials in Honduras. J Nutr 2001;131:262-7.

6. WHO, UNICEF. Complementary feeding of young children in developing countries: a review of current scientific knowledge. Geneva: WHO; 1998.

7. World Health Organization. Complementary feeding: family foods for breastfed children. Geneva: WHO; 2000.

8. Prado MS, Assis AM, Freitas MC, Silva RC, Varjão ML. Padrão e seleção de alimentos complementares e sucedâneos do leite materno em comunidades rurais no semi-árido baiano. Rev Nutr 1995;8:47-64.

9. Nejar FF, Segall-Corrêa AM, Rea MF, Vianna RP, Panigassi G. Padrões de aleitamento materno e adequação energética. Cad Saude Publica 2004;20: 64-71.

10. Venancio SI, Escuder MM, Kitoko P, Rea MF, Monterio CA. Frequência e determinantes do aleitamento materno em municípios do Estado de São Paulo. Rev Saude Publica 2002;36:313-8.

11. Oliveira LP, Assis AM, Pinheiro SM, Prado MS, Barreto ML. Alimentação complementar nos primeiros dois anos de vida. Rev Nutr 2005;18:459-69.

12. Carvalho MS, Andreozzi VL, Codeço CT, Barbosa MT, Shimakura SE. Estimação não-paramétrica: estimador de Kaplan-Meier. In: Carvalho MS, Andreozzi VL, Codeço CT, Barbosa MT, Shimakura SE, editores. Análise de sobrevida: teoria e aplicações em saúde. Rio de Janeiro: Fiocruz; 2005 p. 95-102.

13. World Health Organization. Global strategy for infant and young child feeding - 54 $4^{\text {th }}$ World Health Assembly. Geneva: WHO; 2001.

14. Brunken GS, Silva SM, França GV, Escuder MM, Venâncio SI. Risk factors for early interruption of exclusive breastefeeding and late introduction of complementary foods among infants in midwestern Brazil. J Pediatr (Rio J) 2006;82:445-51.

15. Departamento de Nutrição da Sociedade de Pediatria de São Paulo. Alimentação da criança nos primeiros anos de vida. Rev Paul Pediatr 1998;16:112-7.

16. Egashira EM. Características da dieta e determinantes proximais do consumo energético nos primeiros dois anos de vida no município de São Paulo [dissertação de mestrado]. São Paulo (SP): USP; 1998.

17. Simon VG, Souza JM, Souza SB. Introdução de alimentos complementares e sua relação com variáveis demográficas e socioeconômicas, em crianças no primeiro ano de vida, nascidas em Hospital Universitário no município de São Paulo. Rev Bras Epidemiol 2003;6:29-38.

18. Vieira GO, Silva LR, Vieira TO, Almeida JA, Cabral VA. Hábitos alimentares de crianças menores de 1 ano amamentadas e não-amamentadas. J Pediatr (Rio J) 2004;80:411-6.

19. Silveira FJ, Lamounier JA. Prevalência do aleitamento materno e práticas de alimentação complementar em crianças com até 24 meses de idade na região do Alto Jequitinhonha, Minas Gerais. Rev Nutr 2004;17:437-47.

20. Moura EFA. Duração do período de aleitamento materno de crianças atendidas em ambulatório de pediatria. J Pediatr (Rio J) 1997;73:106-10.

21. Saldiva SR, Escuder MM, Mondini L, Levy RB, Venancio SI. Feeding habits of children aged 6 to 12 months and associated maternal factors. J Pediatr (Rio J) 2007;83:53-8.

22. Tabai KC, Carvalho JF, Salay E. Aleitamento materno e a pratica de desmame em duas comunidades rurais de Piracicaba-SP. Rev Nutr 1998;11: 173-83.

23. Volponi CC, Moura EC. Determinantes do desmame precoce no distrito noroeste de Campinas. Rev. Nutr 2005;18:311-9. 